\title{
Design of On-line Temperature Measurement System for High Voltage Switch Cabinet
}

\author{
Yang Guang $^{1 \mathrm{a}}$, Ji Hao ${ }^{2 b}$, Liu Yangyang ${ }^{1 \mathrm{c}}$, Li Changchun ${ }^{1 \mathrm{~d}}$ \\ ${ }^{1}$ Electric Power Science \& Research Institute of State Grid Tianjin Electirc Power \\ Company, Tianjin,China, 300384 \\ ${ }^{2}$ State Grid Tianjin Electirc Power Company, Tianjin, China, 300010

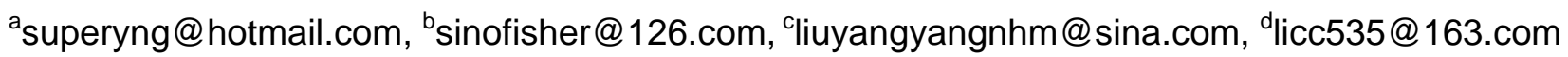

Keywords: Switch cabinet; Optical fiber; Temperature measurement

\begin{abstract}
One of the important equipments in substation switchgear and under high load condition, often appear contact temperature is too high, reliable power supply directly affects the user, or even serious accidents. Based on the comprehensive analysis of the current commonly used temperature measurement technology, and optical fiber sensor as the main research object, based on Fiber Bragg grating switch cabinet on-line temperature measuring system and in the practical application of the system were test effectiveness. The results showed that the optical fiber temperature sensor with high precision, effective for a switch cabinet temperature online monitoring, the staff to control the real-time operation state of the switch cabinet, timely detection and treatment of hidden faults, to offer an important guarantee to ensure the safe operation of the switch cabinet.
\end{abstract}

\section{Introduction}

$10 \mathrm{kV}$ high voltage switchgear is important power equipment in the substation, which plays a role in the distribution of power and load. With the increase of load, switch cabinet, contacts, connectors and other parts of the heating problem is becoming more and more serious, directly affects the user power supply reliability, or even a major security incidents and need of equipment within the switch cabinet temperature constant monitoring[1][2]. But because of the switch cabinet interior space is narrow, closed, low efficiency of traditional artificial inspection method, operation difficulty big, has been difficult to adapt to the practical engineering. Therefore, seeking an effective online automatic measuring system is a problem widely concerned by researchers.

Through comparison of temperature measurement technology, according to the problem of online temperature measurement technology for switchgear, on-line temperature measurement system for switchgear based on fiber grating is designed in this paper [3]. At the same time, it is tested for the actual demand of the fiber grating temperature measurement technology, and according to the actual demand of a substation, the actual test of the system is carried out.

\section{Measurement technique analysis}

Infrared temperature measurement technology. By means of the contact principle of infrared radiation at different temperatures for different, focusing the optical device in equipment of measuring points, collecting the measuring point thermal infrared radiation, again by a photoelectric conversion system will measure the infrared radiation value conversion into electrical signals, after conversion transformation for temperature of the object to be measured values. The infrared temperature measurement technology has the high accuracy and has great influence on the detection accuracy, and it is hard to realize the on-line monitoring and controlling.

Wireless temperature measurement technology. The wireless temperature measurement is an improvement of the contact temperature measurement technology. The data receiver is positioned to distance switch cabinet in a certain distance, between distributed measurement nodes and data receiver by wireless data acquisition and transmission, can be real-time monitoring of the high voltage switch cabinet contact temperature. 
Fiber optic temperature measurement technology. Fiber optic temperature measurement belongs to contact temperature measurement. When the light emitted from the light after modulation, through the optical fiber to reach the sensor, each a reflection sensor to a temperature corresponding to the optical pulse signal, signal processing part of the returned signal filtering sampling and analysis, to measure the temperature of each sensor. High accuracy of fiber temperature measurement [4], has good anti-interference ability.

\section{Study on temperature measurement technology of fiber}

Characteristics of optic fiber sensor. Compared with other sensors, fiber optic sensor has the following advantages:

1. As the sensing signal is basically free from outside electromagnetic field, can be used for long-term reliable continuous on-line detection.

2. Fiber optic sensor has a strong high - pressure performance, suitable for monitoring the safety and production parameters in electric power and other hazardous situations.

3. Small volume, light weight, suitable for switchgear and other equipment temperature monitoring.

Temperature measurement principle. The grating period is usually less than 1 micron fiber grating called fiber Prague grating (FBG), sensing principle is as follows:

When the incident light through the FBG[5][6], meet the conditions of the Bragg wave will be reflected back, through the detection of optical fiber reflective spectrum, you can obtain the corresponding temperature information, so as to realize according to the size of the wavelength of light to choose. As shown in Figure 1.

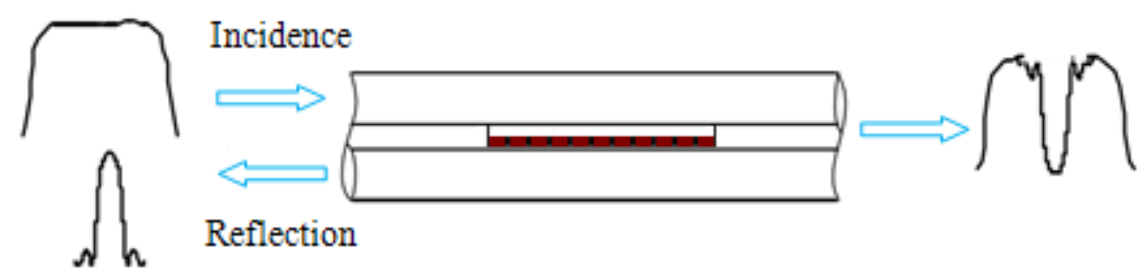

Fig.1 temperature measurement principle of fiber grating

The formula of central wavelength is:

$$
\lambda_{B}=2 n_{\text {eff }} \Lambda
$$

Where:

$n_{\text {eff }}$-Effective index of grating; $\Lambda$-Grating period.

The change of temperature makes the fiber optic strain, so that the effective refractive index and modulation period of the fiber optic are changed, which determines the size of the center wavelength of the fiber. The relationship between central wavelength and temperature change is:

$$
\Delta \lambda_{B}=\lambda_{B}(\alpha+\beta) \Delta \mathrm{T}
$$

Where:

$\Delta \mathrm{T}$ - Temperature change; $\alpha$-Thermal expansion coefficient of fiber;

$\beta$-Thermal optical coefficient; $\Delta \lambda_{B}$ - Wavelength variation.

The relationship between the center wavelength and temperature of the fiber is shown in Figure 2. 


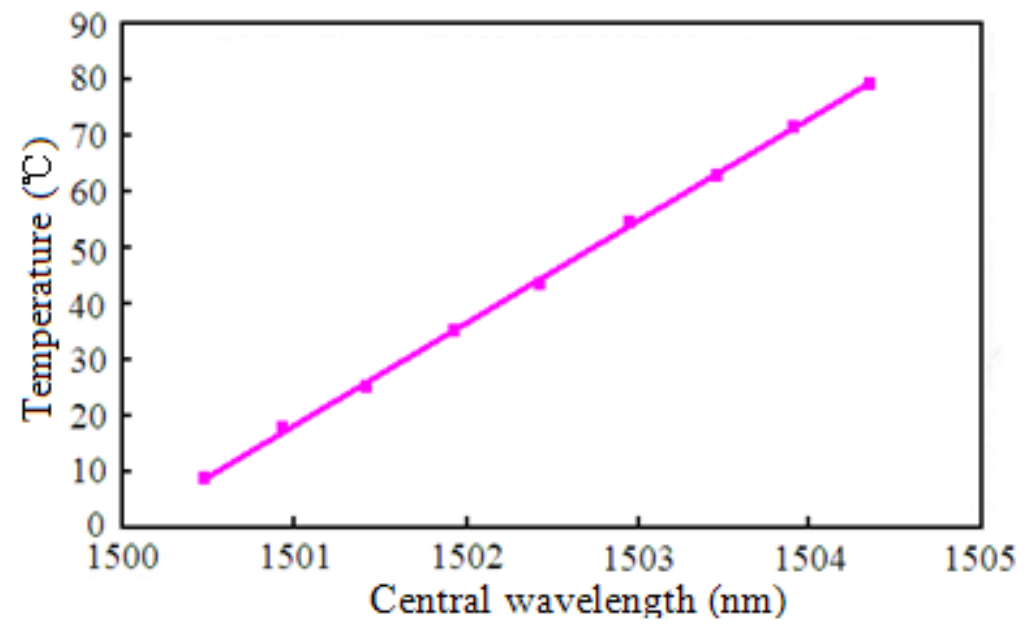

Fig.2 relationship between central wavelength and temperature

\section{Design of temperature measurement system for switchgear based on optical fiber}

Overall scheme design. According to the actual needs, design system overall plan [7]. The whole system can be divided into three levels from the structure: data acquisition layer, station monitoring layer, application management, as shown in Figure 3.

1. Data acquisition layer including grating sensor, optical branching box. The main completion of the measurement points of the data acquisition and optical transmission.

2. Station monitoring layer is composed of PC. Mainly to complete the data analysis, processing, data display, sound and light alarm, etc., the duty room staff can be real-time online viewing of the temperature of multi switch cabinet junction.

3. The application management is mainly for the remote management, so that it can view the monitoring temperature of the computer monitoring data.

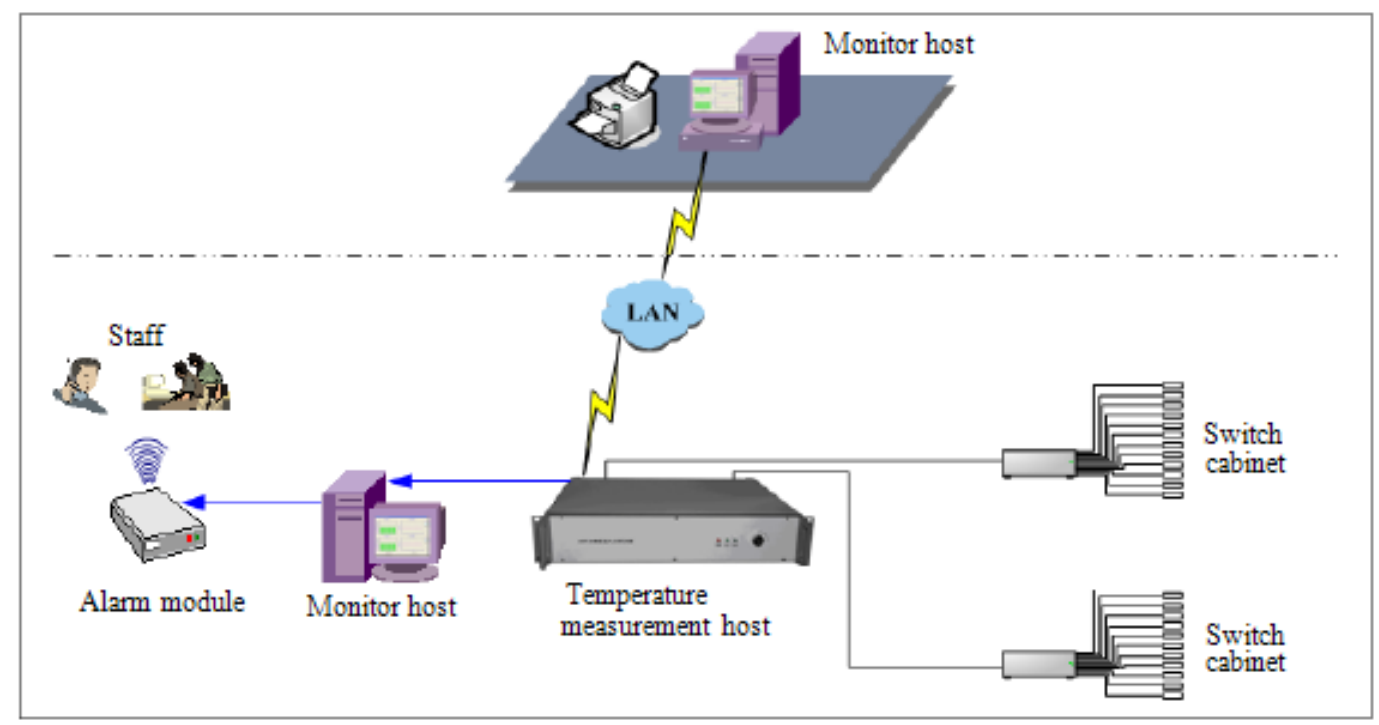

Fig.3 overall structure of temperature measurement system

In the form of direct contact, the fiber optic sensor is installed in the switch cabinet and the position of the temperature is required. Optical fiber sensor receiving spectra emitted from the measurement host. After Fiber Grating Reflective returns the host, when the temperature changes, caused by changes in the wavelength of reflection, so as to realize according to the purpose of measuring wavelength switch cabinet contact temperature.

Temperature measurement host is the data processing center of the temperature measurement system, which is responsible for the transmission, collection and processing of optical signal. 
Temperature inside the host is provided with a pulse signal generation and detection of the original, can receive the reflected modulated signal, for sampling and analysis, determine the temperature every fiber Bragg grating sensor. At the same time, the temperature measurement host also carries on the function which the monitor hosts and the alarm module link.

Function of temperature monitoring system. Terminal monitoring software in the whole system plays a very important role, which is responsible for system configuration entry and system data display, user access database management window, including user management, real-time display module, historical data query module and network communication module, as shown in Figure 4.

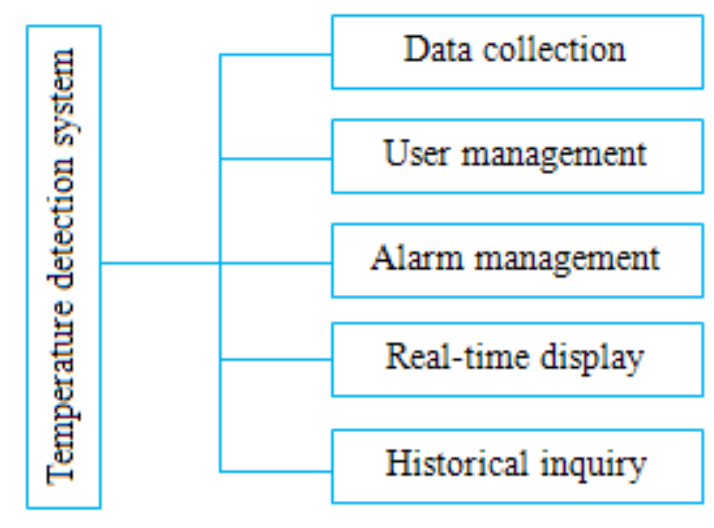

Fig.4 Function of temperature measurement system

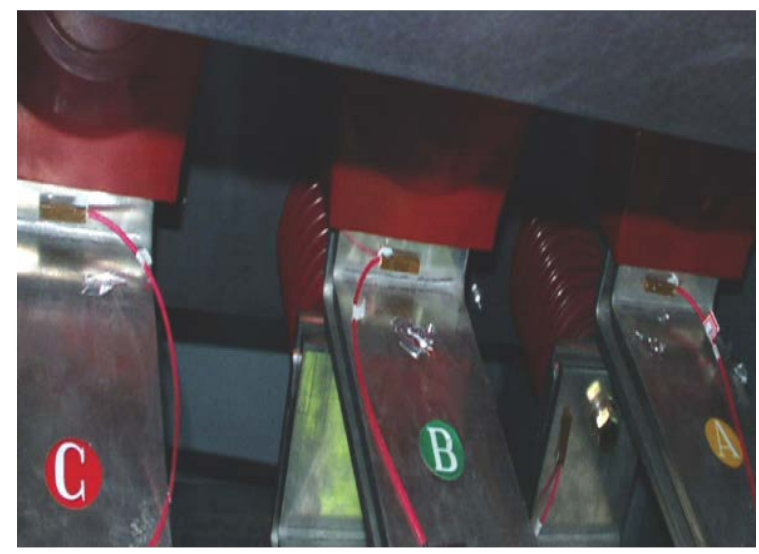

Fig.5 actual installation of optical fiber sensor

\section{Case analysis}

In a substation as the research object, according to the actual configuration of switch cabinet, switch cabinet tested, each switch cabinet placed three optical fiber sensor, sensor grid zone length is $10 \mathrm{~mm}$, the reflectivity was more than $90 \%$, and the installation method of as shown in Figure 5.

In order to verify the accuracy of the results of the fiber optic sensor temperature measurement, the temperature of the contact is measured manually using infrared thermometer and compared with the temperature measured by the fiber optic sensor, as shown in Table 1.

Tab. 1 contact temperature measurement

\begin{tabular}{cccc}
\hline Number of points & Artificial measurement value $\left({ }^{(} \mathrm{C}\right)$ & Fiber optic measurement value $\left({ }^{(} \mathrm{C}\right)$ & Error $\left({ }^{\mathrm{C}}\right)$ \\
\hline 1 & 21.5 & 21.3 & 0.2 \\
2 & 23.6 & 23.9 & -0.3 \\
3 & 19.8 & 20.3 & -0.5 \\
4 & 22.1 & 21.7 & 0.4 \\
5 & 20.5 & 21.0 & -0.5 \\
6 & 19.6 & 19.5 & 0.3 \\
7 & 22.5 & 22.4 & 0.1 \\
8 & 21.2 & 21.6 & -0.4 \\
9 & 22.9 & 22.6 & 0.3 \\
10 & 30.8 & 30.3 & 0.5 \\
11 & 28.6 & 28.2 & 0.4 \\
12 & 29.7 & 29.8 & -0.1 \\
\hline
\end{tabular}

In order to make a better representation of the temperature relationship, the data in Table 1 is represented in Figure 6. 


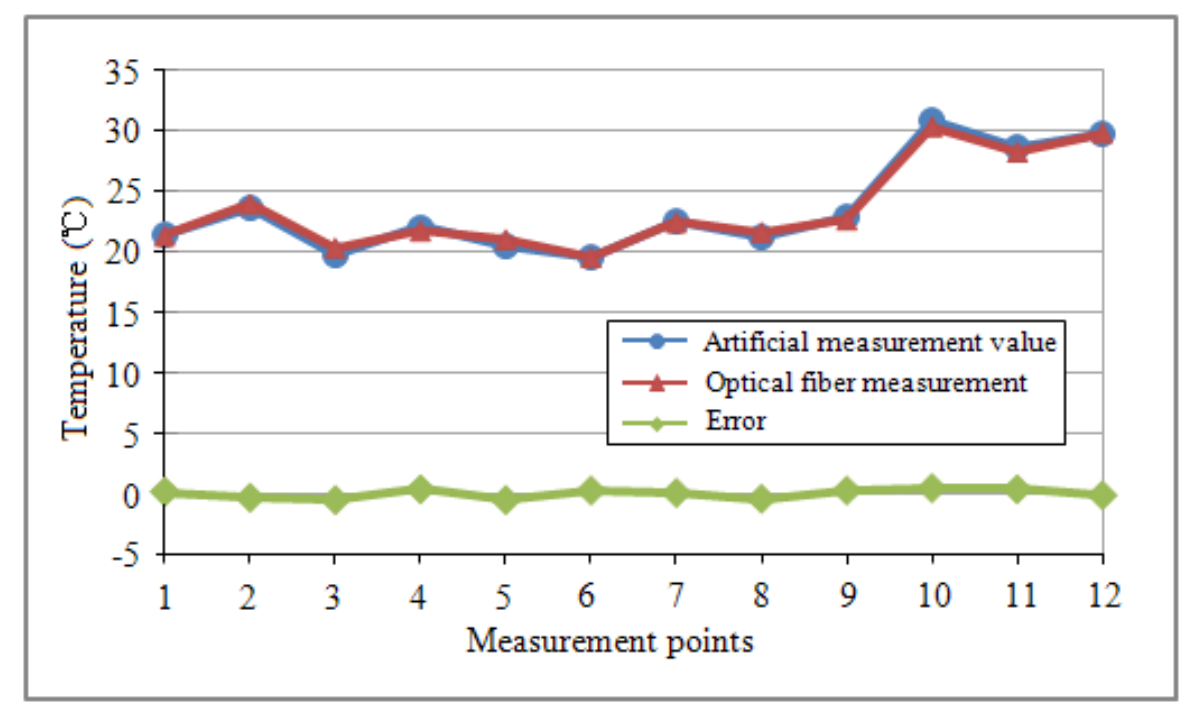

Fig.6 temperature contrast curve

The results show that the temperature measurement of the grating is very close to the actual temperature measurement, and the absolute value of the maximum error is 0.5 . It can be seen that the temperature measurement system using grating sensor can reflect the actual temperature of the switch cabinet, and meet the design requirements.

\section{Conclusion}

The $10 \mathrm{kV}$ High voltage switch cabinet on-line temperature demand as the background, through the analysis of all kinds of measurement techniques, focusing on analysis of the fiber sensor technology, and on the basis of the actual work of the substation design based on Fiber Bragg grating switch cabinet on-line temperature measuring system. The system can effective measure contact the real-time temperature, temperature measurement, high precision, switch cabinet temperature on-line monitoring and real-time control of switch cabinet running state to provide data to support, improve work efficiency, the timely detection and treatment of hidden faults, reduce accidents to ensure the safe operation of high voltage switch cabinet and the whole power system has important engineering significance.

\section{References}

[1] Lee, Byoungho. Review of the present status of optical fiber sensors [J]. Optical Fiber Technology, 2003,9(2):57-79

[2] Christine C. Fibre-optic-based sensors bring new capabilities to structural monitoring[J].Sensor Review.2006,26(3):236-243

[3] Renaud P E.Introduction to ClientlServer systems[J]. John Wiley\&Sons Inc. 2004,34(3):1113

[4] L. Zhen-yu,Y Gen-xing ,C. Li-zhi. Software Test Case Generation with Adequacy Analysis on Scenario-Based Test [J]. Journal of Donghua University. 2011,28(2):139-144

[5] Kobert B. Structural integrity monitoring with fiber Bragg grating sensors[J].Sensor Review.2005, 25(2):78-83

[6] Huang Xiping, Wang Xin, Qiang Wen. Development of On-line Monitoring Equipment of MediumVoltage Switchgear [J].The Eighth International Conference on Electronic Measurement and Instruments, 2007, (3): 388-392. 